\title{
Comparison of Virtual Machine Scheduling Algorithms in Cloud Computing
}

\author{
Karan D. Prajapati \\ Computer Science \& \\ Engineering \\ Nirma University, \\ Ahmedabad, India
}

\author{
Pushpak Raval \\ Computer Science \& \\ Engineering \\ Nirma University, \\ Ahmedabad, India
}

\author{
Miren Karamta \\ Project Scientist \\ BISAG, Gandhinagar, \\ India
}

\author{
M. B. Potdar, Ph.D \\ Project Director \\ BISAG, Gandhinagar, \\ India
}

\begin{abstract}
Cloud Computing is emerging as the next generation platform which would facilitate the user on pay as you use mode as per requirement. The primary aim of Cloud Computing is to provide efficient access to remote and geographically distributed resources with the help of Virtualization in Infrastructure as a Service (IaaS). We need different kind of virtual machines (VM) as per the requirement and cloud provider provides these services as per the Service Level Agreement (SLA) to ensure QoS. For managing large amount of VM requests, the cloud providers require an efficient resource scheduling algorithm. In this paper, a comparative study has been made for different types of VM scheduling and provisioning algorithms and are briefly discussed and analyzed. Then we can conclude that one of these algorithms is batter for scheduling and provisioning with the perspective of cost and security of VMs.
\end{abstract}

\section{General Terms}

Cloud Computing, Virtual Machine, Scheduling, Secure Provisioning, Resource Management

\section{Keywords}

Cloud Computing, VM Scheduling and Provisioning, Resource Overuse, User's perspective, Trust between VMs

\section{INTRODUCTION}

Cloud computing has grown out of developments in grid computing, virtualization and web technologies. National Institute for Standard Technology (NIST) [4] defines cloud computing is a pay-per-use model for enabling available, convenient, on-demand network access to a shared pool of configurable computing resources that can be rapidly provisioned and released with minimal management effort or service provider interaction. Cloud Computing offers subscription based access to Infrastructure, Platforms, and Applications that are popularly referred to as IaaS (Infrastructure as a Service), PaaS (Platform as a Service), and SaaS (Software as a Service) that helps business organizations, academic institutions, government organizations in cutting down operational expenses. The significant features of Cloud Computing include lower cost, incremental scalability, reliability and fault tolerance, serviceoriented, utility-based, virtualization and SLA [5].

Cloud Computing environments can be built on different system infrastructures as per the Service Oriented Architecture(SOA) define like on physically located grid based, Geographically distributed services with large Data Centers (service based), Business based commercial Cloud Computing infrastructure, Educational based collaborative cloud computing etc.

Cloud enables on-demand model for provisioning of computational resources, in the form of virtual machines
(VMs) deployed in a cloud provider's geographically distributed datacenters. As per the define requirements from the customer side computational resources are shared among users according to the Pay-Per-Use model. The allocation of resource (i.e. VMs, Applications etc) and proper scheduling has significant impact on the performance of the system. The primary aim of CC is to provide efficient access to remote and geographically distributed resources that can be given with the proper infrastructure. An efficient Scheduling, Provisioning, Load Balancing, Security aware infrastructure is needed to manage the access to the different resources. There are different types of resource scheduling in Cloud that are based on different parameters like cost, performance, resource utilization, time, priority, and physical distances, through put, bandwidth, and resource availability. In this paper we have concentrate on the Cost effective, Energy efficient and security aware scheduling and provisioning algorithms. If we need commercial cloud then Cost effective and Energy efficient cloud environment is needed. If some organization need secure data transmission and sharing of secure remote application then we have to think about secure scheduling and provisioning that do not affect the neighbor resources and prevent side-channel attack and DOS attack of one VM to the other VMs and PMs.

\section{VM SCHEDULING}

Scheduling is a balancing scenario in which processes or tasks are scheduled as per the given requirements and used algorithm. In Cloud Computing VM scheduling algorithms are used to schedule the VM requests to the Physical Machines (PM) of the particular Data Center (DC) as per the requirement fulfilled with the requested resources (i.e. RAM, Memory, Bandwidth etc). In today's era there are so many cloud providers in market that have different capacity of Data Centers and Physical Machines available. SalesForce, Amazon, Microsoft office 365 and Windows Azure, Oracle Cloud, Google Apps etc are leading cloud providers of the year of 2013 [6]. In general scheduling algorithm works in three levels as per given below [2]:

1. For the set of VMs find the appropriate Physical Machine.

2. Determine the proper provisioning scheme for the VMs.

3. Scheduling the tasks on the VMs.

Mainly scheduling algorithms are described as static or dynamic algorithms. First-Come-First-Severed (FCFS) is a good example of static VM scheduling algorithm. Genetic Algorithm is a good example of dynamic VM scheduling algorithm that will be discuss later in the next section. Eucalyptus uses greedy or Round Robin algorithm, with GREEDY the first node which can meet the initial requirements will be chosen. The OpenNebula default scheduler provides a rank scheduling policy that places VMs 
on physical machines as per the rank of PMs.

Cloud provisioning is also important factor that comes in managing the cloud resources in cost effective, energy efficient or secure aware manner as discussed in Introduction section. Cloud Provisioning consists of three steps: (1) Virtual Machine Provisioning, (2) Resource Provisioning and (3) Application Provisioning [7]. Here, we have to concentrate on Virtual Machine Provisioning.

\section{VM SCHEDULING ALGORITHMS}

In this section we describe the VM scheduling and provisioning algorithms, those are used for optimization of different factors like Time, Cost, Energy and Security. There are very less algorithms are available that provision the VMs with the perspective of the security of neighboring VMs or nodes.

\subsection{Round Robin}

The Round Robin algorithm focuses on distributing the load equally to all the nodes. Using this algorithm, the Scheduler allocates one VM to a node in a cyclic manner. The round robin scheduling for VM scheduling is similar to the round robin scheduling for process scheduling. The scheduler starts assigning VM to each node and move further for next VM to place in to next node. This algorithm is repeated for all the nodes until each node have at least on VM. After placing VMs to all nodes it will go to the first node and repeat this process for next VMs requests. Hence, in this case, the scheduler does not wait for the exhaustion of the resources of a node before moving on to the next. As an example, if there are three nodes and three VMs are to be scheduled, each node would be allocated one VM, provided all the nodes have enough available resources to run the VMs.

The main advantage of this algorithm is that it utilizes all the resources in a balanced order. An equal number of VMs are allocated to all the nodes which ensure fairness. However, the major drawback of using this algorithm is that the power consumption will be high as many nodes will be kept turned on for a long time. If three resources can be run on a single node, all the three nodes will be turned on when Round Robin is used which will consume a significant amount of power. The CloudSim [9] toolkit supports RR scheduling strategy for internal scheduling of jobs.

\subsection{Genetic Algorithm}

Genetic algorithms are stochastic search algorithms based on the mechanism of natural selection strategy. It starts with a set of initial solution, called initial population, and will generate new solution using genetic operators. The genetic algorithm approach computes the impact in advance, that it will have on the system after the new VM resource is deployed in the system, by utilizing historical data and current state of the system. It then picks up the solution, which will have the least effect on the system [10].

The advantage of this technique is it can handle a large searching space, applicable to complex objective function and can avoid trapping by local optimum solution. Authors of [11] have developed a cost-based job scheduling algorithm, which provide a multi QoS scheduling in cloud computing environment.

\section{Match-Making Algorithm}

The MMA (match-making algorithm), algorithm first filter out the nodes or hosts those do not meet the VM requirements and do not have enough resources (Like CPU, Memory,
Processors etc) to place and run the VM. Rank will be given to nodes as per the gathered information by the monitoring drivers. If any variable comes in monitoring then it will be included in to rank expression [13]. The result of rank expression is given to the cloud scheduler and monitoring driver makes decision for VMs placement and reconfiguration.

OpenNebula has default match making scheduler that implements the Rank scheduling policy. OpenNebula comes with Haizea Scheduler that support advance reservation of resources and queuing of best effort requests [3]. The goal of this algorithm is to prioritize resources those are most suitable for the VM. Those resources with a higher rank are used first to allocate VMs.

\section{A Trust Aware Distributed and Collaborative Scheduler}

The Trust Aware Distributed and Collaborative Scheduler (TADCS) present a scheduler for VM placement and provisioning in clouds. It is based on $p 2 p$ architecture allowing a fully de- centralized model. This scheduling algorithm makes decisions based on dynamic-resources centric algorithm that computes a score and on user's objectives (i.e. Processing Units, Memory, Disk Space, Bandwidth etc). The score is evaluated based on static and dynamic resources usage but also uses the resources quota associated with each vm. Resources quotas are of two types: (1) Soft Quota: Quantity of resources dedicated to the VM and, (2) Hard Quota: Quantity of resources used by the VM that is not dedicated to particular VM . The user's objectives ease the expression of quality of trust and protection and the deployment of HPC architecture. These objectives are given by the user for each of his/her VMs [12].

This algorithm is perfect algorithm for secure provisioning of resources that separate the adversary VMs from the legitimate VMs from the same Physical Machine and migrate to the other PM or Data Center. Only cost effective or Energy efficient solution is not proper strategy form the user's perspective. We have to think about the secure resource management that does not affect the QoS that comes with the SLA agreement. To give secure solution with the neighboring VMs we need proper scoring functions that can be calculated based on how much times the VM is migrated because of overuse of the resources of other VMs and can be optimized from the existing Trust Aware Scheduler algorithm.

\subsection{Memory-Aware Cloud Scheduling}

In memory-aware scheduling, the scheduler collects the information of cache behavior of each VM from each physical machine, and migrate VMs that can potentially reduce the overall cache misses and the average memory access latencies by NUMA affinity in the cloud system. In each PM, a monitor checks (Last-Level Cache) LLC/L3 cache misses with hardware performance monitoring counters, and periodically sends the per-VM LLC miss and NUMA affinity information to the cloud scheduler. Based on the VM status information from all the nodes, the cloud scheduler makes global scheduling decisions. [14] Cache-Aware scheduler that work in two phase local (scheduling based on cache behavior of one node) and global (scheduling based on cache behavior of all nodes), and NUMA-Aware scheduler that work in global phase only. These memory aware schedulers reduce the memory contentions between VMs from the same host, but number of migrations will increase. 


\section{COMPARISION OF ALGORITHMS}

Determination of best scheduling algorithm for cloud computing is depends on various factors. For scheduling and provisioning of resources different algorithms are available that are aware of particular factor. We have comparison of such algorithms with factors like Time, Power, Cost, Security and Memory. Into that we are concentrating on the Trust Aware Distributed and Collaborative Scheduler that is comparatively batter than the others. This algorithm can suspend the adversary VM at the time of over use of resources. So, to optimize the trust aware scheduler will be our next research work.

\begin{tabular}{|l|c|c|c|c|c|}
\hline Algorithm & $\begin{array}{c}\text { Time } \\
\text { Efficient }\end{array}$ & $\begin{array}{c}\text { Power } \\
\text { Aware }\end{array}$ & $\begin{array}{l}\text { Cost } \\
\text { Effective }\end{array}$ & Aware & $\begin{array}{c}\text { Memory/ } \\
\text { Bandwidth } \\
\text { Aware }\end{array}$ \\
\hline Round- & $\checkmark$ & - & - & - & - \\
\hline Genetic & - & $\checkmark$ & $\checkmark$ & - & - \\
\hline Algorithm & & - & $\checkmark$ & - & - \\
\hline $\begin{array}{l}\text { Match- } \\
\text { Making }\end{array}$ & - & & & & \\
\hline $\begin{array}{l}\text { TADCS } \\
\text { Memory } \\
\text {-Aware }\end{array}$ & - & - & - & $\checkmark$ & $\checkmark$ \\
\hline
\end{tabular}

\section{CONCLUSION}

In Cloud computing environment heterogeneous resources are provided as services in forms of virtual machines that should be managed in optimized way with efficient scheduling. In this study, various scheduling algorithms with the perspective of Cost effective, Energy efficient and Security aware are studied. To solve the resource scheduling problem various scheduling algorithms based on various factors have been tried by various researchers. In to that there is a lack of security aware scheduling and provisioning algorithms in cloud computing. So, this paper put some sine on the security perspective resource management in cloud environment.

\section{FUTURE WORK}

Future work will include new scheme of secure scheduling and provisioning that will be implemented in open source cloud environment. That proposed algorithm will be given to set of Physical Machine (i.e. Data Center) and set of Data Centers (i.e. Cloud) and results will be compared.

\section{ACKNOWLEDGEMENT}

Authors would like to thanks T. P. Singh, Director, Bhaskaracharya Institute for Space Applications \& GeoInformatics and Prof. Sharada Valiveti, Nirma University for their constant encouragement for this research work.

\section{REFERENCES}

[1] C T Lin, "Comparative Based Analysis of Scheduling Algorithms for Resource Management in Cloud Computing Environment", Vol. 1, Issue-1, July 2013.

[2] Marc E. Frincu, St'ephane Genaud and Julien Gossa, "Comparing Provisioning and Scheduling Strategies for Workflows on Clouds", 2013.

[3] http://opennebula.org/documentation:archives:rel2.0:sch g accessed on Oct 302013.

[4] P. Mell and T. Grance, The NIST Definition of Cloud Computing, National Institute of Standards and Technology, Information Technology Laboratory, Technical Report Version 15, 2009.

[5] G. Boss, P. Malladi, D. Quan, L. Legregni and H. Hall, Cloud Computing (White Paper), IBM, october2007, http://download.boulder.ibm.com/ibmdl/pub/software/dw /wes/hipods/Cloud_computing_wp_final_8Oct.pdf accessed on Oct 30, 2013.

[6] http://talkincloud.com/talkin039-cloud-top-100 cloudservices-providers/top-100-cloud-servicesproviders-csps-2013-list-unv accessed on Nov 92013.

[7] Rodrigo N. Calheiros, Rajiv Ranjan, and Rajkumar Buyya, "Virtual Machine Provisioning Based on Analytical Performance and QoS in Cloud Computing Environments", 40th International Conference on Parallel Processing (ICPP) IEEE Computer Society, 2011, pp.295-304 DOI: 10.1109/ICPP.2011.17

[8] Shin-ichi Kuribayash",Optimal Joint Multiple Resource Allocation Method for Cloud Computing Environments", International Journal of Research and Reviews in Computer Science (IJRRCS) Vol. 2, No. 1, March 2011.

[9] Rodrigo N. Calheiros, Rajiv Ranjan, Anton Beloglazov, Cesar A. F. De Rose and Rajkumar Buyya, "CloudSim: a toolkit for modeling and simulation of cloud computing environments and evaluation of resource provisioning algorithms", Published online 24 August 2010 in Wiley Online Library (wileyonlinelibrary.com). DOI: 10.1002/spe.995.

[10] J. Yu and R. Buyya, "Scheduling Scientific Workflow Applications with Deadline and Budget Constraints using Genetic Algorithms", Scientific Programming Journal, 14(3-4), 217-230, IOS Press, 2006.

[11] D Dutta, R C Joshi ,"A Genetic -Algorithm Approach to Cost-Based Multi-QoS Job Scheduling in Cloud Computing Environment", International Conference and Workshop on Emerging Trends in Technology (ICWET 2011) - TCET, Mumbai, India,2011

[12] J. Rouzaud-Cornabas, "A trust aware distributed and collaborative scheduler for virtual machines in cloud", 2011.

[13] GWDG eScience Group, "Virtual Machine Allocation in Current Cloud Computing Middleware", 2012.

[14] Jeongseob Ahn, Changdae Kim, Jaeung Han, Young-ri Choi, and Jaehyuk Huh, "Dynamic Virtual Machine Scheduling in Clouds for Architectural Shared Resources", 2011. 